\title{
Coil Formation in Multishell Carbon Nanotubes: Competition between Curvature Elasticity and Interlayer Adhesion
}

\author{
Ou-Yang Zhong-can, and Zhao-Bin Su \\ Institute of Theoretical Physics, Chinese Academy of Sciences, \\ P.O. Box 2735, Beijing 100080, China \\ Chui-Lin Wang \\ China Center of Advanced Science and Technology (World Laboratory), \\ P.O. Box 8730, Beijing 100080, China
}

(January 24, 2018)

\begin{abstract}
To study the shape formation process of carbon nanotubes, a string equation describing the possible existing shapes of the axis-curve of multishell carbon tubes (MCTs) is obtained in the continuum limit by minimizing the shape energy, that is the difference between the MCT energy and the energy of the carbonaceous mesophase $(\mathrm{CM})$. It is shown that there exists a threshold relation of the outmost and inmost radii, that gives a parameter regime in which a straight MCT will be bent or twisted. Among the deformed shapes, the regular coiled MCTs are shown being one of the solutions of the string equation. In particular, the optimal ratio of pitch $p$ and radius $r_{0}$ for such a coil is found to be equal to $2 \pi$, which is in good agreement with recent observation of coil formation in MCTs by Zhang et al.

61.48.+c, 68.65.+g, 68.70.+w
\end{abstract}

Typeset using REVTEX 
Since the discovery of straight and multishell carbon nanotubes (MCTs) in arc discharges [1], many unique and novel properties have been predicted for the tubes. Among them, an especially intriguing one is their structural stability, the mechanical properties of MCTs are expected to be significantly stiffer than any presently known materials [2]. However, in the recent synthesis by the catalytic decomposition of gas such as acetylene, a significant fraction of the produced MCTs exhibits various curved shapes [3], of which the most striking shapes are helices, $i$. e., the regular coils. It has been pointed out that the tubes can be twisted and deformed by an abrupt release of energy and a singularity in the stress-strain curve [4]. In Ref. [3], the regular coil formation was explained by a periodic distribution of pentagonheptagon-pair dislocations (PHPDs), but why the PHPDs are distributed periodicly rather randomly still remains as an open question. Dunlap pointed out that the regularity could be caused by defect-defect interactions and constraints on the optimal heptagon-pentagon nanotube bend [5]. In Ref. [6], the bent carbon tubes were simulated by classical molecular dynamics based upon the three-body Tersoff-Brenner interatomic potential, but it is rather difficult to extract the essential physics from such a numerical approach.

Therefore, the general questions can be posed as follows, what is the mechanism of the curved deformation for the MCTs, and can we derive the deformed shape.

In this Letter, we analytically obtain the general equilibrium-shape equation of the axiscurve of the MCT in the continuum limit by taking account of competition among the curvature elasticity, the adhesion of the interlayer van der Waals bonding and the tension of the outer and inner surfaces of a MCT. The sum of these three energies can be understood as the shape formation energy (see bellow for details). We find that the variation of the shape formation energy yields an equation of rigid string, which has been studied extensively in differential geometry [7]. Straight line and regular coil are two exact solutions of the equation. We show that under certain geometric conditions the shape formation energy of a straight MCT could become negative, in other words, the straight MCT becomes unstable in a quench-like formation process, and as a result, bent or twisted MCTs will be formed spontaneously to keep the equilibrium condition ( $i$. e. the zero shape formation energy). 
Taking into consideration of the equilibrium condition in the quench-like cooling processes, the above argument provides an insight for the mechanism of the curved deformation for the MCTs. The optimal ratio of the pitches and radii of the regular coils formed in such processes is shown impressively to be equal to $2 \pi$. Our result is in good agreement with recent observation of the coil formation in MCTs by Zhang et al. [3].

Generally, when the hydrocarbons are thermally decomposited [3], the carbon molecules are condensed mostly to form an isotropic smectic-like crystal, $i$. e. the carbonaceous mesophase (CM), while the remained space is filled by plate-like molecules [8]. Thus the MCT formation is quite similar to the tube formation of a smectic- $A$ phase grown from isotropic phase in liquid crystal [9]. As shown in Ref. [9], the shape formation energy is the additional energy of an MCT with respect to $\mathrm{CM}$, which is a sum of the following three terms, (i) the net difference of the volume free energy between MCT and CM, i.e. $F_{V}=-g_{0} V$ where $V$ is the volume of the MCT and $-g_{0}$ is the adhesion energy density of the interlayer van der Waals bonding, (ii) the surface energy $F_{A}=\gamma\left(A_{o}+A_{i}\right)$ where $\gamma$ is the surface tension, $A_{o}$ and $A_{i}$ are the areas of the outmost and inmost surfaces, respectively, and (iii) the curvature elastic energy of the layers.

We consider first the third term of the shape formation energy, the curvature elastic energy. MCTs can be treated as a set of curved graphite layers [1]. For a single layer, the curvature elastic energy is an increment part of the in-layer covalent energy due to the layer curvature. Following Lenosky et al. [10], the curvature elastic energy of a single layer curved graphite carbon has the form as,

$$
E_{b}^{(s)}=\epsilon_{1} \sum_{i}\left(\sum_{<j>} \mathbf{u}_{i j}\right)^{2}+\epsilon_{2} \sum_{<i, j>}\left(1-\mathbf{n}_{i} \cdot \mathbf{n}_{j}\right)+\epsilon_{3} \sum_{<i, j>}\left(\mathbf{n}_{i} \cdot \mathbf{u}_{i j}\right)\left(\mathbf{n}_{j} \cdot \mathbf{u}_{j i}\right),
$$

where $\mathbf{u}_{i j}$ is a unit vector pointing from carbon atom $i$ to its neighbour $j$ and $\mathbf{n}_{i}$ is a unit vector normal to the fullerene surface at atom $i$. The summation $\sum_{<j>}$ is taken over the three nearest neighbour $j$ atoms to atom $i$, and the sums of the last terms are taken over only the nearest neighbour atoms. The superscript $(s)$ emphasizes the energy $E_{b}$ is for a single layer. Our first task is to reduce Eq. (1) into a continuum form. 
A curved single-shell tube of radius $\rho$ without inclusion of its two end-caps can be described by

$$
\mathbf{Y}(s, \phi)=\mathbf{r}(s)+\rho(\mathbf{N}(s) \cos \phi+\mathbf{b}(s) \sin \phi)
$$

where $0<\phi<2 \pi$, and $0<s<l$ is the arc-length parameter along the curved tube-axis whereas curve $\mathbf{r}(s)$ is the vector representation for the curve of the tube axis. $l$ is the total length of the tube. $\mathbf{N}(s)$ and $\mathbf{b}(s)$ are the unit normal and unit binormal vectors of $\mathbf{r}(s)$ respectively [11]. Making use of the well-known Frenet formulas [11],

$$
\mathbf{t}_{s}=k(s) \mathbf{N}, \quad \mathbf{N}_{s}=-k(s) \mathbf{t}-\tau(s) \mathbf{b}, \quad \mathbf{b}_{s}=\tau(s) \mathbf{N},
$$

where $\mathbf{t}=d \mathbf{r} / d s, \mathbf{t}_{s}=d \mathbf{t} / d s, \mathbf{N}_{s}=d \mathbf{N} / d s, \mathbf{b}_{s}=d \mathbf{b} / d s, k(s)$ and $\tau(s)$ are the curvature and torsion of $\mathbf{r}(s)$ respectively. In ref. [12], we have derived the area element $d A=\rho(1-$ $\rho k \cos \phi) d \phi d s$, the mean curvature $H=(2 \rho k \cos \phi-1) / 2 \rho(1-\rho k \cos \phi)$ and the Gaussian curvature $K=-k \cos \phi / \rho(1-\rho k \cos \phi)$ for the tube surface of $\mathbf{Y}$. It is then easy to prove $\oint K d A=0$. According to the Gauss-Bonnet theorem [11], this means the topology of the curved tube is the same as that of a straight tube. From the Euler's theorem in topology, the surface can be perfectly embedded by a carbon network of six-member rings as in plane graphite layer. With the help of Frenet formula (3), we transform the vector functions in Eq. (1) into continuum limit by expanding them up to the order of $O\left(a^{2} k^{2}\right)$, where $a=1.42 \AA$ is the bond length in the graphite layer,

$$
\mathbf{u}_{i}(M)=\mathbf{u}_{i j}=\left(1-\frac{a^{2}}{6} k^{2}(M)\right) \mathbf{t}(M)+\left(\frac{a}{2} k(M)+\frac{a^{2}}{6} k_{s}(M)\right) \mathbf{N}(M)-\frac{a^{2}}{6} k(M) \tau(M) \mathbf{b}(M),
$$

where $k_{s}=d k / d s, M=1,2,3$ denote three families of $s p^{2}$-bonded curves with one curve of each family acrossing from atom $i$ to its three neighbour atoms $j$ on the surface where the carbon atoms embedded, and have one to one correspondence to atoms $j$. Here we would like to emphasize that all the expressions $K(M), t(M), N(M), \tau(M)$, and $b(M)$ are functions of the arc-length $s$, where $s=i a$. Specifying the tube surface described by Eq. (2), in 
which the $s p^{2}$-bonded curves can be considered approximately as geodesies for the curved surface $\mathbf{Y}$, we have additionally $\mathbf{N}(M)=\mathbf{n}_{i}, k(M)=c_{1} \cos ^{2} \theta(M)+c_{2} \sin ^{2} \theta(M), \tau(M)=$ $\left(c_{1}-c_{2}\right) \sin \theta(M) \cos \theta(M)$ where $c_{1}$ and $c_{2}$ are the two principal curvatures of the surface at atom $i$ location, $i$.e., $H=\left(c_{1}+c_{2}\right) / 2$ and $K=c_{1} c_{2}$, and $\theta(M)$ are the angles between $c_{1}$ direction and $\mathbf{t}(M)$. Considering $\sum_{M=1}^{3} \sin ^{2} \theta(M)=3 / 2$ and $\sum_{M=1}^{3} \sin ^{4} \theta(M)=9 / 8$, and substituting Eq. (4) into Eq. (1), we obtain an important formula for the curvature elastic energy of the tube [12,

$$
E_{b}^{(s)}=\oint\left[\frac{1}{2} k_{c}(2 H)^{2}+\bar{k} K\right] d A
$$

where the bending elastic constant,

$$
k_{c}=(1 / 32)\left(18 \epsilon_{1}+24 \epsilon_{2}+9 \epsilon_{3}\right)\left(a^{2} / \sigma\right)
$$

with $\sigma=\sqrt{3} a^{2} / 4=2.62 \AA^{2}$ being the occupied area per atom, and the saddle-splay modulus is,

$$
\bar{k}=-\left(8 \epsilon_{2}+2 \epsilon_{3}\right) k_{c} /\left(6 \epsilon_{1}+8 \epsilon_{2}+3 \epsilon_{3}\right) .
$$

Formula (5) is actually a general expression of the elastic energy which is valid also to fluid membranes [13] and solid shells [14]. If we substitute $\left(\epsilon_{1}, \epsilon_{2}, \epsilon_{3}\right)$ in Eqs. (6-7) by the values of $(0.96,1.29,0.05) \mathrm{eV}$ respectively, which were calculated by Lenosky et al. using a local density approximation [10], we find $k_{c}=1.17 \mathrm{eV}$ and $k_{c} / \bar{k}=-1.56$. The obtained value of $k_{c}$ is in reasonable agreement with the value of $1.02 \mathrm{eV}$ calculated by Tersoff [15] using an atomistic method for straight tubes, and is excellently close to the value of $1 . .2 \mathrm{eV}$ extracted from the measured phonon spectrum of graphite [16]. The calculated ratio of $k_{c} / \bar{k}$ is also close to the result of $k_{c} / \bar{k}=-105.4 / 88=-1.2$ measured by Blakeslee et al. [17]. Therefore, we have sufficient confidence on Eq. (5). Moreover, since it has been averaged over three nearest neighbours for each site in expression Eq. (1) of Lenosky et al. [10], we can have only two invariants as $H^{2} d A$ and $K d A$ in Eq. (5). Therefore, within the same approximation, the free energy $F_{b}^{(s)}$ corresponding to Eq. (5) should be again a linear combination of these 
two invariants only. Consequently, $F_{b}^{(s)}$ would have the same formal expression as Eq. (5) with coefficient $k_{c}$ and $\bar{k}$ being temperature dependent.

To extend the above result to a MCT, one has to integrate Eq. (5) from its inmost radius $\rho_{i}$ to the outmost radius $\rho_{o}$. We may apply a similar treatment as that of [18] which is in fact devoted to the curved smectic crystal multilayers, and has a layer structure similar to graphites'. Replacing $k_{11}$ with $k_{c} / d$ in Eq. (3) of Ref. [18 and neglecting the constant term associated with $\oint K d A$, we have

$$
F_{b}=\sum F_{b}^{(s)}=\left(k_{c} / 2 d\right) \oint 2 \sqrt{H^{2}-K} \ln \left(\frac{1-D H+D \sqrt{H^{2}-K}}{1-D H-D \sqrt{H^{2}-K}}\right) d A,
$$

where $d=3.4 \AA$ is the space between two neighbour graphite layers and $D=\rho_{o}-\rho_{i}$ is the thickness of the MCT. Here, the surface integral is carried out over the inner surfaces. Using the above expressions for $H, K$, and $d A$ and integrating from $\phi=0$ to $2 \pi$, we obtained the curvature elastic energy for the MCT as

$$
F_{b}=\left(\pi k_{c} / d\right) \int\left[\ln \left(\frac{\rho_{o}}{\rho_{i}}\right)+\ln \left(\frac{1+\sqrt{1-k^{2} \rho_{i}^{2}}}{1+\sqrt{1-k^{2} \rho_{o}^{2}}}\right)\right] d s
$$

We now turn to consider the other two terms of the shape formation energy, $F_{V}$ and $F_{A}$, both of which are weak binding energy, $i . e$. the adhesion energy between layers of an MCT. Despite the fact that many of the structural properties of plane graphites are well understood, the calculation of interlayer adhesion energy for curved graphites is still an open question. The observation in Ref. [1] reveals that the interlayer distance $d$ in MCTs remains to be the same as that in plane graphite, but the in-layer lattice structures for each singleshell tube in one MCT may have different helicity. In other words, the interlayer lattices are not in perfect registry (referred to "incommensurate" or "mismatched" lattices). Therefore, the attractive forces between layers in an MCT cannot be accounted for by conventional forces in plane graphites [19]. However, the adhesion energy for mismatched lattices is often smaller than that for commensurate surfaces. We use a mismatched parameter $\eta$ to take account for the mismatched effect between the interlayer lattices, $0<\eta \leq 1$, and $\eta=1$ 
corresponds to the commensurate case. Since we are so far not aware of more detailed knowledges, as the lowest approximation, we take the following simple energy form,

$$
F_{V}+F_{A}=-g_{0} \pi\left(\rho_{0}^{2}-\rho_{i}^{2}\right) \int d s+2 \pi \gamma\left(\rho_{0}+\rho_{i}\right) \int d s
$$

where $-g_{0}=\eta \Delta E_{c} / d, \Delta E_{c}=-330 \mathrm{erg} / \mathrm{cm}^{2}=-2.04 \mathrm{eV} / \mathrm{nm}^{2}$ is the interlayer cohesion energy of planar graphite obtained theoretically by Girifalco and Lad [19], and $\gamma$ is the tension of the outmost and inmost surfaces of the MCT, which is equal to half of the energy needed to separate two unit-area surfaces, $i . e ., \gamma=-(1 / 2) \Delta E_{c}$.

Usually we have the MCTs with $\rho_{o}^{2} k^{2}<<1$, then the expression $\ln \left[\left(1+\sqrt{1-k^{2} \rho_{i}^{2}}\right) /(1+\right.$ $\left.\left.\sqrt{1-k^{2} \rho_{o}^{2}}\right)\right]$ can be approximated to $(1 / 4)\left(\rho_{o}^{2}-\rho_{i}^{2}\right) k^{2}$, and the shape formation energy of the MCT, Eqs. (9-10), can be subsequently simplified to,

$$
F=F_{V}+F_{A}+F_{b}=m \int d s+\alpha \int k^{2} d s
$$

where $m=\pi\left(k_{c} / d\right) \ln \left(\rho_{o} / \rho_{i}\right)+2 \pi \gamma\left(\rho_{0}+\rho_{i}\right)-\pi g_{0}\left(\rho_{o}^{2}-\rho_{i}^{2}\right)$ and $\alpha=(1 / 4) \pi\left(k_{c} / d\right)\left(\rho_{o}^{2}-\rho_{i}^{2}\right)$. Eq. (11) is nothing but a string action [20]. The variational equation $\delta F=0$ yields the equilibrium-shape equations of the string [7],

$$
\begin{gathered}
2 k_{s s}+k^{3}-2 k \tau^{2}-\frac{m}{\alpha} k=0 \\
k^{2} \tau=\text { const. }
\end{gathered}
$$

where $k_{s s}=d^{2} k(s) / d s^{2}$.

Following what has been discussed for the derivation of curvature elastic energy, in the derivation for these three terms of the shape formation energy, we have expressed all the relevant quantities in terms of geometric language. Therefore, due to the reason of geometric symmetry on the low dimensional manifolds, the corresponding free energy should have the same expression as those of the derived shape formation energy with the coefficients becoming temperature dependent. We will keep such understand in the above and hereafter discussions. 
It is obvious that a straight line is always a solution of the string equations (12) and (13), since its $k$ and $\tau$ are zero. The corresponding shape formation energy of a straight MCT is $F=m l$. The shape formation energy is regarded as a free energy and the equilibrium threshold condition of $F=0$ yields the criteria for the growth of a straight MCT as

$$
m=\pi\left(k_{c} / d\right) \ln \left(\rho_{o} / \rho_{i}\right)+2 \pi \gamma\left(\rho_{o}+\rho_{i}\right)-\pi g_{0}\left(\rho_{o}^{2}-\rho_{i}^{2}\right)=0
$$

This equation describes the geometric relation between $\rho_{o}$ and $\rho_{i}$ in terms of the physical parameters $k_{c}, \gamma$, and $g_{0}$ for a straight MCT. Both $\gamma$ and $g_{0}$ are also dependent on the formation temperatures and catalyst. So the detailed data measured from the produced MCTs can reveal the properties of $\gamma$ and $g_{0}$ with the help of Eq. (14). The formation procedure for MCTs, either a quick growth in which the temperature can be regarded as constant or a sudden cooling, is actually a sort of quench-like process. As long as the shape formation energy for the straight MCTs, i. e., deviates downwards from the threshold condition in the formation procedure, becomes negative, the resultant remnant part of energy will prevent the straight MCT from keeping stable. Then a shape deformation will be induced and it would lead to another solution of the string equation with its shape formation again being equal to zero. Therefore, by considering the threshold condition $F=0$, any outward growth by increasing $\rho_{0}$ will make the straight MCT undergo a shape deformation as long as $g_{0}$ being kept constant. Furthermore, $g_{0}$ may increase with temperature decreasing, following again Eq. (14), a straight MCT grown may also be coiled under the cooling process. These features give a natural explanation for the deformation of MCTs.

Now we would prove that the observed MCT regular coils shown in Ref. [3] are just the allowed solutions of equations (12) and (13). Mathematically, the regular coils can be described by vectors,

$$
\mathbf{r}(s)=\left(r_{0} \cos \omega s, r_{0} \sin \omega s, h \omega s\right)
$$

where the coiled pitch $p=2 \pi h, r_{0}$ is the coil radius and $R \equiv \omega^{-1}=\sqrt{r_{0}^{2}+h^{2}}$. One can easily show from the Frenet formulas, Eq. (3), that $k=\omega^{2} r_{0}, \tau=-\omega^{2} h$, and the regular coil curves are the solutions of (12) and (13) if their $r_{0}$ and $h$ satisfy the following equation: 


$$
r_{0}^{2}-2 h^{2}-\frac{m}{\alpha}\left(r_{0}^{2}+h^{2}\right)^{2}=0
$$

Introducing $h / R=\sin \theta$, and $r_{0} / R=\cos \theta$, and taking into account of Eq. (16), we have $R^{2}=(\alpha / m)\left(\cos ^{2} \theta-2 \sin ^{2} \theta\right)$, and $k^{2}=r_{0}^{2} / R^{4}=(m / \alpha)\left[1 /\left(1-2 \tan ^{2} \theta\right)\right]$. Therefore, the coil formation energy can be derived from (11) as

$$
F=m l\left[1+\frac{1}{1-2 \tan ^{2} \theta}\right]
$$

Since now we have the coil situation which is quite different from the straight MCT case, even for the negative value of $m$ in Eq. (17), we may treat the threshold condition $F=0$ as $\tan ^{2} \theta=h^{2} / r_{0}^{2}=1$ or

$$
\frac{p}{r_{0}}=2 \pi
$$

We compare the optimal ratio given by Eq. (18) with the experimental results reported in Ref. [3] and find a good agreement. As shown in Fig. 1 of Ref. [3], there is a fraction (about 10\%) of MCTs being regularly coiled with a variety of radii $r_{0}$ and helix pitches p. By a direct evaluation from the figure, we do find $p / r_{0}=2 \pi$ is well hold. A rough estimation from the coil shown in the inset of this figure gives $p \approx 600 \mathrm{~nm}$ and $r_{0} \approx 100$ nm, i. e. $p / r_{0} \approx 6 \approx 2 \pi$. Another coil, shown in Fig. 2 of the same reference, has its $p \approx 700 \mathrm{~nm}$ and $r_{0} \approx 100 \mathrm{~nm}$ which leads to $p / r_{0} \approx 7$, again close to the prediction of Eq. (18). Moreover, the results corresponding to the coil are shown in Fig. 3 of Ref. [3], $\tan \theta=\frac{\pi}{2}-\phi_{0} \approx 2.2 / 2 \approx 1.1$, are also in good agreement with the present prediction of $\tan \theta=1$, where $\phi_{0}$ is defined within the context of Ref. [3].

It is also interesting to study the value of $m$ for the MCT coil by utilizing the data provided in Ref. [3], where the experimentally observed values of $2 \rho_{i}=3 \sim 7 \mathrm{~nm}$ and $2 \rho_{o}=15 \sim 20 \mathrm{~nm}$. Making use of calculated values, $k_{c}=1.17 \mathrm{eV}$ and $g_{0}=\eta \times 2.04 \times$ $10^{-2}\left(\mathrm{eV} / \AA^{2}\right) / d=6.03 \eta \mathrm{eV} / \mathrm{nm}^{3}$, we can estimate $\pi g_{0}\left(\rho_{o}^{2}-\rho_{i}^{2}\right)=(1.0 \eta \sim 1.7 \eta) \times 10^{3} \mathrm{eV} / \mathrm{nm}$, and $\pi\left(k_{c} / d\right) \ln \left(\rho_{o} / \rho_{i}\right)+2 \pi \gamma\left(\rho_{o}+\rho_{i}\right)=68 \sim 101 \mathrm{eV} / \mathrm{nm}$. Considering expression (14), we find that under reasonable approximation, $m$ in the practically formed MCT coils always 
take negative value. Such a fact is notably consistent with the above proposed explanation for the shape deformation mechanism of MCTs.

In summary, by deriving a string action type expression for the formation energy of the MCTs as well as its equilibrium-shape equation, we have shown that there is a threshold condition for the formation of straight MCTs, below that the straight MCTs become unstable and will undergo a shape deformation. In particular, we derive further an optimal formation condition $p / r_{0}=2 \pi$ for the regular coil solution which is in good agreement with the recent experiment observations.

This work is partly supported by the National Natural Science Foundation of China. 


\section{REFERENCES}

[1] S. Iijima, Nature 354, 56 (1991); S. Iijima, T. Ichihaschi, and Y. Ando, Nature 356, $776(1992)$.

[2] M. S. Dresselhaus, Nature, 358, 195 (1992).

[3] X. B. Zhang et al. , Europhys. Lett. 27, 141 (1994).

[4] B. I. Yakobson, C. J. Brabec, and J. Bernholc, Phys. Rev. Lett. 76, 2511 (1996).

[5] B. I. Dunlap, Phys. Rev. B50, 8134 (1994).

[6] C. J. Brabec et al. , in APS March Meeting, APS Bull. 40, 420 (1995).

[7] J. Langer and D. A. Singer, J. Diff. Geom. 20, 1 (1984).

[8] J. L. White, Prog. in Solid State Chem. , 9, 59 (1975) ; J. E. Zimmer and J. L. White, Mol. Cryst. Liq. Cryst. , 38, 177 (1977); K. Kinoshita, Carbon: Electrochemical and Physicochemical Properties (John Wiley \& Son, New York, 1988) p. 25-27.

[9] H. Naito, M. Okuda, and Ou-Yang Zhong-can, Phys. Rev. Lett. 70, 2912 (1993).

[10] T. Lenosky et al. , Nature, 355, 333 (1992).

[11] M. P. do Carmo, Differential Geometry of Curves and Surfaces ( Prentice-Hall, New Jersey, 1976).

[12] The detail will appear in a full paper by the present authors.

[13] W. Helfrich, Z. Naturforsch, 28c, 693 (1973).

[14] L. D. Landau and E. M. Lifshitz, Theory of Elasticity, 3rd ed. (Pergamon, Oxford, 1986)

[15] J. Tersoff, Phys. Rev. B46, 15546 (1992).

[16] R. Nicklow, N. Wakabayashi, and H. G. Smith, Phys. Rev. B5, 4951 (1972).

[17] O. L. Blakeslee et al. J. Appl. Phys. 41, 3373 (1970), see, also D. J. Srolovitz, S. A. 
Safran, and R. Tenne, Phys. Rev. E49, 5260 (1994).

[18] H. Naito, M. Okuda, and Ou-Yang Zhong-can, Phys. Rev. E52, 2095 (1995).

[19] L. A. Girifalco and R. A. Lad, J. Chem. Phys. 25, (1956).

[20] A. M. Polyakov, Nucl. Phys. B286, 406 (1986).

[21] S. Ihara, S. Itoh, and J. Kitakami, Phys. Rev. B48, 5643 (1993). 